\title{
Strong Convergence in Stabilised Degenerate Convex Problems*
}

\author{
Wolfgang Boiger $^{1, * *}$ and Carsten Carstensen ${ }^{1, * * *}$ \\ ${ }^{1}$ Humboldt-Universität zu Berlin, Institut für Mathematik, Unter den Linden 6, D-10099 Berlin
}

\begin{abstract}
Solutions to non-convex variational problems typically exhibit enforced finer and finer oscillations called microstructures such that the infimal energy is not attained. Those oscillations are physically meaningful, but finite element approximations typically experience dramatic difficulty in their reproduction. The relaxation of the non-convex minimisation problem by (semi-)convexification leads to a macroscopic model for the effective energy. The resulting discrete macroscopic problem is degenerate in the sense that it is convex but not strictly convex. This paper discusses a modified discretisation by adding a stabilisation term to the discrete energy. It will be announced that, for a wide class of problems, this stabilisation technique leads to strong $H^{1}$-convergence of the macroscipic variables even on unstructured triangulations. This is in contrast to the work [2] for quasi-uniform triangulations and enables the use of adaptive algorithms for the stabilised formulations.
\end{abstract}

(c) 2008 WILEY-VCH Verlag GmbH \& Co. KGaA, Weinheim

\section{Introduction}

Variational problems with non-(quasi-)convex energy densities develop finer and finer oscillations and have no classical solution in general. Macroscopic models can be achived by convexifying the energy. Since such problems are usually not strictly convex, minimisation algorithms may encounter singular Hessian matrices. A remedy are stabilisation techniques. Such methods have been proposed in [2], where it is proven that proper stabilisation can yield strong $H^{1}$-convergence on quasiuniform triangulations. We will suggest a stabilisation technique which yields $H^{1}$-convergence even on non quasi-uniform triangulations.

Our model problem is defined as follows: Let $\Omega \subset \mathbf{R}^{n}$ be a bounded Lipshitz domain with polygonal boundary, $n=2,3$, $p \geq 2$ and $m \in \mathbf{N}$. The set of admissible functions is $\mathcal{A}:=V+u_{D}$, where $V=W_{0}^{1, p}\left(\Omega ; \mathbf{R}^{m}\right)$, and the Dirichlet conditions are given by $u_{D} \in W^{1, p}\left(\Omega ; \mathbf{R}^{m}\right) \cap C\left(\bar{\Omega} ; \mathbf{R}^{m}\right)$ with $\left.u_{D}\right|_{\partial \Omega} \in H^{2}\left(E ; \mathbf{R}^{m}\right)$ for all edges or faces $F$ of $\partial \Omega$.

With a smooth convex energy density $W^{* *}: \mathbf{R}^{m, n} \rightarrow \mathbf{R}$ and some convex lower order term $L: \mathcal{A} \rightarrow \mathbf{R}$, the (convex) minimisation problem reads

$$
\text { minimise } \quad E(v):=\int_{\Omega} W^{* *}(\nabla v) \mathrm{dx}+L(v) \quad \text { amongst } v \in \mathcal{A} .
$$

Solutions are equivalently characterised by the corresponding Euler-Lagrange equations (see [3]): We denote $S(X):=$ $D W^{* *}(X)$ for $X \in \mathbf{R}^{m, n}$ and $J(v ; w):=D L(v ; w)$ for $v, w \in V$, then the Euler-Lagrange equations consist in finding $u \in \mathcal{A}$ with

$$
\int_{\Omega} S(\nabla u): \nabla v \mathrm{dx}+J(u ; v)=0 \quad \text { for all } v \in V,
$$

where $X: Y$ is the scalar product on $\mathbf{R}^{m, n}$.

\section{Discretisation \& Stabilisation}

In our efforts towards the discretisation of (1), we assume $\left(\mathcal{T}_{\ell}\right)_{\ell \in \mathbf{N}_{0}}$ to be a shape-regular family of triangulations of $\Omega$ in the sense of Ciarlet, and $\mathcal{F}_{\ell}^{\Omega}$ the set of inner edges or faces of $\mathcal{T}_{\ell}$. We denote the diameter of an element $T \in \mathcal{T}_{\ell}$ with $h_{T}:=\operatorname{diam}(T)$ as well as $h_{F}:=\operatorname{diam}(F)$ for $F \in \mathcal{F}_{\ell}^{\Omega}$, and $H_{\ell}:=\max _{T \in \mathcal{T}_{\ell}} h_{T}$. Let $u_{D, \ell} \in \mathcal{S}^{1}\left(\mathcal{T}_{\ell} ; \mathbf{R}^{m}\right)$ be the nodal interpolation of $u_{D}$, where $\mathcal{S}^{1}\left(\mathcal{T}_{\ell} ; \mathbf{R}^{m}\right)$ is the lowest order conforming finite element space defined on $\mathcal{T}_{\ell}$ containing $\mathbf{R}^{m}$ valued functions, and $\mathcal{A}_{\ell}:=V_{\ell}+u_{D, \ell}$, where $V_{\ell}=V \cap \mathcal{S}^{1}\left(\mathcal{T}_{\ell} ; \mathbf{R}^{m}\right)$.

We modify the energy term by adding a positive semi-definite symmetric stabilisation term

$$
a_{\ell}: H^{2}\left(\mathcal{T}_{\ell} ; \mathbf{R}^{m}\right) \times H^{2}\left(\mathcal{T}_{\ell} ; \mathbf{R}^{m}\right) \rightarrow \mathbf{R},
$$

\footnotetext{
* Supported by DFG Research Center MATHEON "Mathematics for key technologies" in Berlin.

** E-mail: boiger@math.hu-berlin.de, Phone: +4930 20935427

*** E-mail: cc@ math.hu-berlin.de, Phone: +49 302093 5489, Fax: +49 3020935444
} 


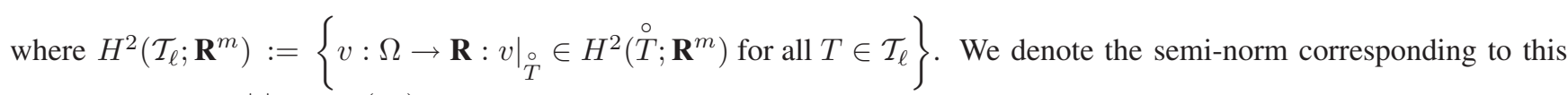
bilinear-form with $|\cdot|_{\ell}:=a_{\ell}(\cdot, \cdot)$. With this stabilisation, the discrete problem reads

$$
\operatorname{minimize} \quad E_{\ell}(v):=E(v)+a_{\ell}(v, v) \text { amongst } v \in \mathcal{A}_{\ell} .
$$

With $J_{\ell}(v ; w):=J(v ; w)+a_{\ell}(v, w)$, the corresponding discrete Euler-Lagrange equations consist in finding $u_{\ell} \in \mathcal{A}_{\ell}$ such that

$$
\int_{\Omega} S\left(\nabla u_{\ell}\right): \nabla v \mathrm{dx}+J_{\ell}\left(u_{\ell} ; v\right)=0 \quad \text { for all } v \in V_{\ell}
$$

\section{Convergence Results}

The subsequent assumptions are similar to [2, (H1)-(H5)].

Suppose there are $\alpha, r, s>0$ with $1<r \leq 2$ and $r p>s+p$ such that for all $X, Y \in \mathbf{R}^{m, n}$ it holds

$$
|S(X)-S(Y)|_{F}^{r} \leq \alpha\left(1+|X|_{F}^{s}+|Y|_{F}^{s}\right)(S(X)-S(Y)):(X-Y)
$$

where $|\cdot|_{F}$ is the norm corresponding to ":". We remark that (2) is implied by

$$
|S(X)-S(Y)|_{F}^{r} \leq \alpha\left(1+|X|_{F}^{s}+|Y|_{F}^{s}\right)\left(W^{* *}(Y)-W^{* *}(X)-S(X):(X-Y)\right) .
$$

Furthermore, denote $e_{\ell}:=u-u_{\ell}$, and suppose there is a constant $M>0$ such that

$$
J(u ; v)-J\left(u_{\ell} ; v\right) \leq M\left\|e_{\ell}\right\|_{L^{2}(\Omega)}\|v\|_{L^{2}(\Omega)} \quad \text { for all } v \in W^{1, p}\left(\Omega ; \mathbf{R}^{n}\right) .
$$

With $\rho_{F}:=\frac{H_{\ell}^{1+\gamma}}{h_{F}}$ for $F \in \mathcal{F}_{\ell}^{\Omega}$ and a fixed $\gamma \in(-1,3)$, we define for $v, w \in H^{2}\left(\mathcal{T}_{\ell} ; \mathbf{R}^{m}\right)$ the stabilisation term

$$
a_{\ell}(v, w):=\sum_{F \in \mathcal{F}_{\ell}^{\Omega}} \rho_{F} \int_{F}[\nabla v]:[\nabla w] \mathrm{ds}_{\mathrm{x}}
$$

where $[\cdot]$ denotes the jump of a function along the an edge or face.

We assume that the continuous solution $u \in \mathcal{A}$ satisfies $u \in W^{2, p}\left(\Omega ; \mathbf{R}^{m}\right)$, and the discrete solutions $u_{\ell}$ are bounded with respect to the $W^{1, p}$ semi-norm independently of $\ell$. Then Lemmas 4.2 and 4.3 and the main Theorem 4.6 in [1] imply the following results.

Theorem 3.1 Let $m \in(0, M)$ such that $m\left\|e_{\ell}\right\|_{L^{2}(\Omega)}^{2} \leq J\left(u ; e_{\ell}\right)-J\left(u_{\ell} ; e_{\ell}\right)$ for all $\ell$. Then we have

$$
\left\|e_{\ell}\right\|_{L^{2}(\Omega)}^{2}+\left|e_{\ell}\right|_{\ell}^{2}=\mathcal{O}\left(H_{\ell}^{\zeta}\right) \quad \text { and } \quad\left\|e_{\ell}\right\|_{H^{1}(\Omega)}^{2}=\mathcal{O}\left(H_{\ell}^{\xi}\right)
$$

where $\zeta=\min \left\{1+\gamma, \frac{r}{r-1}\right\}$, and $\xi=\min \left\{\frac{1+\gamma}{2}, \frac{r}{r-1}-\frac{1+\gamma}{2}\right\}$.

Theorem 3.2 Assume $0 \leq J\left(u ; e_{\ell}\right)-J\left(u_{\ell} ; e_{\ell}\right)$ for all $\ell$ and let $z \in \boldsymbol{R}^{2}, z \neq 0$ be a constant vector such that $\left\|z \cdot \nabla e_{\ell}\right\|_{L^{2}(\Omega)}^{2} \leq C_{z} \int_{\Omega} \delta_{\ell}: \nabla e_{\ell} \mathrm{dx}$ holds with a constant $C_{z}>0$ independently from $\ell$. Then we have

$$
\left\|e_{\ell}\right\|_{L^{2}(\Omega)}^{2}+\left|e_{\ell}\right|_{\ell}^{2}=\mathcal{O}\left(H_{\ell}^{\zeta}\right) \quad \text { and } \quad\left\|e_{\ell}\right\|_{H^{1}(\Omega)}^{2}=\mathcal{O}\left(H_{\ell}^{\xi}\right)
$$

where $\zeta=\min \{1+\gamma, 2\}$, and $\xi=\min \left\{\frac{1+\gamma}{2}, 2-\frac{1+\gamma}{2}\right\}$.

Acknowledgements The first author WB thankfully acknowledges the support of the Deutsche Forschungsgemeinschaft (DFG) through the RTG 1128.

\section{References}

[1] W. Boiger, C. Carstensen: On the Strong Convergence of Gradients in Stabilised Degenerate Convex Minimisation Problems, in preparation.

[2] S. BARTEls, C. CARstensen, P. Plecháč, A. Prohl: Convergence for stabilisation of degenerately convex minimisation problems, IFB 6, 2004, pp. 253-269.

[3] B. Dacorogna: Direct Methods in the Calculus of Variations, Springer, Berlin, 1989. 